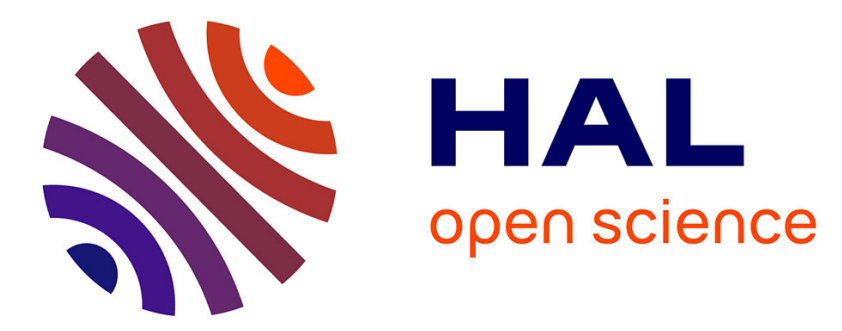

\title{
Precursor Shear Elastic Pseudo-Isotropy in Near Second Order Martensitic Phase Transitions
}

\author{
H. Chopra, M. Wuttig
}

\section{To cite this version:}

H. Chopra, M. Wuttig. Precursor Shear Elastic Pseudo-Isotropy in Near Second Order Martensitic Phase Transitions. Journal de Physique IV Proceedings, 1995, 05 (C8), pp.C8-157-C8-160. 10.1051/jp4:1995819 . jpa-00254068

\section{HAL Id: jpa-00254068 https://hal.science/jpa-00254068}

Submitted on 1 Jan 1995

HAL is a multi-disciplinary open access archive for the deposit and dissemination of scientific research documents, whether they are published or not. The documents may come from teaching and research institutions in France or abroad, or from public or private research centers.
L'archive ouverte pluridisciplinaire HAL, est destinée au dépôt et à la diffusion de documents scientifiques de niveau recherche, publiés ou non, émanant des établissements d'enseignement et de recherche français ou étrangers, des laboratoires publics ou privés. 


\title{
Precursor Shear Elastic Pseudo-Isotropy in Near Second Order Martensitic Phase Transitions
}

\author{
H.D. Chopra and M. Wuttig \\ Department of Materials and Nuclear Engineering, The University of Maryland, College Park, \\ MD 20742-2115, U.S.A.
}

\begin{abstract}
In contrast to the behavior of the isentropic elastic constants both isothermal constants, $C^{\prime}$ and $\mathrm{C}_{44}$, of In-Cd, In-Tl and Fe-Pd alloys soften on approaching the martensite start temperature. The isothermal, i.e. low frequency, $\approx 500 \mathrm{~Hz}$, anisotropy ratio approaches a value of unity as the martensitic transformation is approached from above. The parent phase becomes shear elastically pseudo-isotropic. Close to the transition, this shear pseudo-isotropy reflects the reorientation of incipient variants in the cubic phase under the influence of the dynamic measuring stress. It represents a true dynamical precursor of this fcc $\Leftrightarrow$ fct near second order martensitic phase transition.
\end{abstract}

\section{INTRODUCTION}

Materials undergoing a martensitic transition (MT) exhibit various behavioral anomalies in their physical properties in a temperature range above, but close to the transition temperature, $\mathrm{M}_{s}$. Across the whole spectrum of systems undergoing MT, steel martensites are strongly first order; shape-memory alloys are moderately first order; the low $\mathrm{T}_{\mathrm{c}}$ A15 compounds $\mathrm{Nb}_{3} \mathrm{Sn}$ and $\mathrm{V}_{3} \mathrm{Si}, \mathrm{In}-\mathrm{Tl}$, and perovskite-ferroelectrics are nearly second order in nature. The alloy systems in the first group have not shown significant precursor effects except for defect

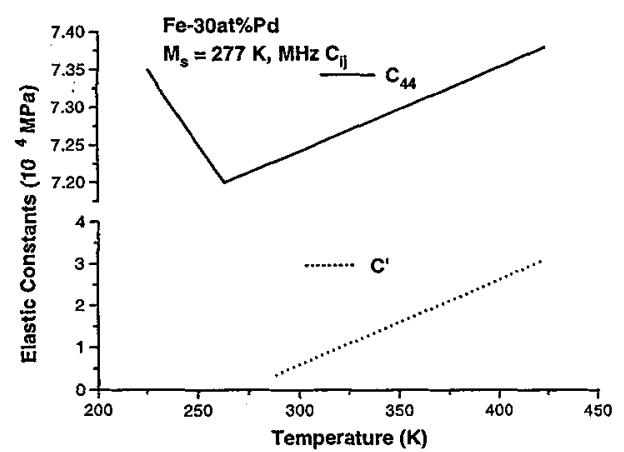

Figure 1: Schematic of the temperature dependence of the $\mathrm{MHz} \mathrm{C}^{3}$ and $\mathrm{C}_{44}$ elastic constants in an $\mathrm{Fe}-30$ at\% $\mathrm{Pd}$ alloy, [12]. related anomalies. However, the second and the third groups exhibit a wide array of anomalies in the premartensitic regime. For instance, the $\beta-W$ compounds $\mathrm{V}_{3} \mathrm{Si}$ [1] and $\mathrm{Nb}_{3} \mathrm{Sn}$ [2] exhibit extreme lattice softening of the shear modulus, $C^{\prime}$. At the same time the phonon softening is incomplete. Recently, by using system-specific models, it has become clear that even with incomplete phonion softening, the product phase is related to the soft mode, and a MT may involve a complex set of static and dynamic modulations leading to the transition [3], [4], [5], [6], [7], [8], [9]. However, a unified approach of looking at these transitions from a generalized point of view has yet to emerge.

In the present study, the model alloy systems of Fe-Pd and $\mathrm{In}-\mathrm{Cd}$ were investigated to better understand 


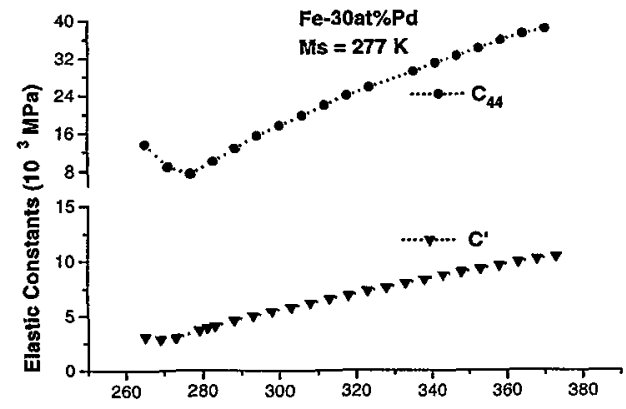

Figure 2: Temperature dependence of the low frequency $C^{\prime}$ and $C_{44}$ elastic constants in an $\mathrm{Fe}-30$ at\%Pd alloy.

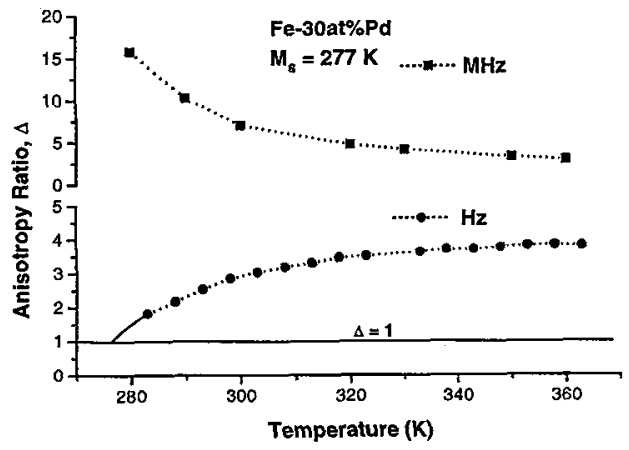

Figure 3: Temperature dependence of the adiabatic and isothermal anisotropy ratio for an $\mathrm{Fe}-30$ at\% $\%$ alloy. the low frequency premartensitic elastic behavior, and to attempt to form a generic explanation to these near second order martensitic phase transitions. Based on this, a new perspective, pretransitional Shear Elastic Pseudo-Isotropy has evolved.

\section{Experimental Details}

Single crystals of Fe-Pd and In-Cd were grown using the modified Bridgeman technique. The boules of Fe-Pd and In-Cd were then annealed at $1473 \mathrm{~K}$ and $425 \mathrm{~K}$ respectively, for a period of one week to remove any chemical inhomogeneity that may have been introduced during their casting or growth. The Laue back-reflection method was used to orient the single crystals in the $\langle 100\rangle_{\mathrm{FCC}}$ and $\langle 110\rangle_{\mathrm{FCC}}$ directions for the low frequency $(<500 \mathrm{~Hz})$ flexural and torsional experiments. The accuracy in orientation was in excess of $0.5^{\circ}$. Suitably oriented crystals were spark-cut into torsional rods of circular cross-section or flexural reeds to prevent any stress to be introduced. The low frequency premartensitic elastic properties of In-Cd and Fe-Pd alloy systems were studied using an apparatus developed by Aning et al. [10]. More details of the present experiments are given elsewhere [11].

\section{Experimental Results}

Figure 1 shows the $\mathrm{MHz}$ premartensitic elastic behavior of an Fe-30at\%Pd alloy system as a function of temperature [12]. It demonstrates that on approaching the transition temperature, C' softens sharply. Even $\mathrm{C}_{44}$ softens slightly, although this decrease is just $2 \%$ over a temperature interval of 100 $\mathrm{K}$ above the transition temperature. Similar behavior is observed in the In-Tl [13] and In-Cd [14] alloy systems at $\mathrm{MHz}$ frequencies in which $\mathrm{C}_{44}$ hardens, however. In contrast to the $\mathrm{MHz}$ premartensitic behavior of these alloys, the low frequency premartensitic elastic behavior of In-Cd and Fe-Pd investigated in the present study, and also the In-TI alloy system [15] showed, in addition to the known C' softening, an anomalous softening of $\mathrm{C}_{44}$ on approaching the transition temperature. This is shown in Fig. 2 for the Fe-30at\%Pd alloy. In addition, these

values of $\mathrm{C}_{44}$ and $\mathrm{C}^{\prime}$, approximately $10^{2}$ to $10^{3} \mathrm{MPa}$, were found to be one to two orders of magnitude smaller than those measured at the $\mathrm{MHz}$ frequencies.

Figure 3 shows the temperature dependence of the $\mathrm{MHz}$ and low frequency anisotropy ratio, $\Delta=\mathrm{C}_{44} / \mathrm{C}^{\prime}$, in the Fe30.0at\%Pd alloy. It can be seen that at $\mathrm{MHz}$ frequencies, this ratio increases sharply, in sharp contrast to the temperature dependence of the low frequency anisotropy ratio behavior for the same alloy, also shown in Fig. 3. At low frequencies, remarkably, the anisotropy ratio decreases rather than increases showing that at low frequencies the material exhibits a tendency to become shear elastically isotropic upon approaching the transition temperature. A ratio $\Delta=1$ would mean ideal isotropy. Since the cubic phase does not become isotropic in the conventional 
sense of the word, the term shear elastic pseudo-isotropy of the transforming cubic phase has been employed, and its significance will be discussed in the next section.

\section{Pseudo-Isotropy In Near Second Order Martensitic FCC $\Leftrightarrow$ FCT Phase Transitions}

As mentioned earlier, the premartensitic state exhibits numerous anomalies. These anomalies, in terms of the crystal- and microstructure, represent the heterogeneous state of the system, preparative to the structural transformation. The tweed structure is a well known example of such a heterogeneity yielding insight into the statics of this state. The experimental results described above yield insight into its dynamics. It is known that the population of martensite variants can be controlled by applying a static stress field during cooling through the transformation. For the present alloys, such a field can lift the 3 -fold degeneracy of the product phase. The shear elastic pseudo-isotropy found here can be understood in much the same way except that now the degeneracy is periodically removed by the application of the dynamic measuring stress.

The situation may also be likened to the determination of elastic constants at constant entropy and temperature. Classically, this difference between the isentropically and isothermally measured elastic constants is caused by the finite time is takes to dissipate the isentropically generated heat. In the vicinity of near second order phase transitions an additional relaxation can occur through the relaxation of the alloy which can lead to a redistribution of incipient variants. A discussion of this effect can be based on the Landau free energy [16]. At temperatures above the phase transition, this energy displays a minimum at the origin representing the stable cubic phase. In addition it displays three lobes directed along the angles $\theta_{1}=0, \theta_{2}=2 / 3 \pi$ and $\theta_{3}=2 \times 2 / 3 \pi$ which, close to the transformation, become metastable states. The isentropic elastic constant $C^{\prime}$ is given by the curvature of the Landau free energy in the direction of these angles. The isentropic elastic constant $\mathrm{C}_{44}$ is determined by the curvature of this energy at the origin but now along a direction halfway between the above angles and is independent of $\mathrm{C}^{\prime}$ as dictated by the cubic symmetry. On the contrary, the isothermal elastic constant $\mathrm{C}_{44}$ is coupled to $\mathrm{C}^{\prime}$. The coupling is facilitated through the relaxation of the potential which at temperatures sufficiently close to $M_{s}$ leads to the redistribution of incipient variants with respect to the applied stress. The relaxation for this process is determined by the anharmionic nature of the Landau free energy and will be discussed in a future publication.

It is thus qualitatively clear that the isothermal behavior of the premartensitic state is governed by the relaxation of the Landau free energy under stress. Close to the transformation temperature, where the product phase is metastable, the relaxation is tantamount to a redistribution of incipient variants. The dynamics of the relaxation will be determined by a relaxation time so that it can be observed at low frequencies and is effectively frozen at higher frequencies, in agreement with the experimental observations [17].

\section{Conclusions}

The single crystal elastic response of $\mathrm{Fe}-\mathrm{Pd}$, In-Cd, and also $\mathrm{In}-\mathrm{Tl}$ alloy systems exhibits a $\mathrm{C}_{44}$ anomaly in addition to the C' anomaly normally observed at $\mathrm{MHz}$ frequencies. Furthermore, the low frequency isothermal elastic coefficients are one to two orders of magnitude smaller than the absolute values of the iserntropic elastic coefficients obtained at $\mathrm{MHz}$ frequencies. Their low frequency anisotropy ratio decreases rather than increases towards the transition temperature and approaches a value of unity at the $M_{s}$ temperature. This anomaly is attributed to the relaxation of the Landau free energy leading to a shear elastically pseudo-isotropic state of the cubic crystal. It represents the real dynamic precursor phenomenon in near second order fec $\Leftrightarrow$ fct martensitic phase transitions.

\section{Acknowledgments}

This study was supported by the Office of Naval Research, contract No. N00014-93-10506 and the National Science Foundation, Grant No. DMR-93-21185, it also benefitted from support by the Army Research Office, contract No. DAAL03-92-G-0121.

\section{References}

[1] Pyte, E., Phys. Rev. Lett. 25, (1970) 1176-1180.

[2] Rehwald, W., Ryal, M., Cohen, R. W., and Cody, G. D., Phys. Rev. B 6, (1972) 363-371.

[3] Clapp, P. C., Phys. Status Solidi B 57, (1973) 561-569.

[4] Suzuki, T., and Wuttig, M., Acta Metall. 23, (1975) 1069-1076.

[5] Shapiro, S. M., Yang, B. X., Noda, Y., Tanner, L. E., and Schryvers, D., Phys. Rev. B 44, (1991) 93019313. 
[6] Shapiro, S. M., Yang, B. X., Shirane, G., Noda, Y., and Tanner, L. E., Phys. Rev. Lett. 62, (1989) 12981301.

[7] Gooding, R. A., and Krumhansl, J. A., Phys. Rev. B 39, (1989) 1535-1540.

[8] Shapiro, S. M., Noda, Y., Fujita, Y., and Yamada, Y., Phys. Rev. B 30, (1984) 4314-4321.

[9] Yamada, Y., Met. Trans A 19A, (1988) 777-787.

[10] Aning, A., Suzuki, T., and Wuttig, M., J. Appl. Phys. 10, (1982) 6797- 6808.

[11] Li, J., Chopra, H. D., Tanner, L. E., and Wuttig, M., "Elastic Isotropy in Martensitically Transforming FCC Alloys", Shape-Memory Materials and Phenomena- Fundamental Aspects and Applications Boston, MA, 3-5 December, C. T. Liu and K. Otsuka Eds. (MRS, Pittsburgh, PA 1991) pp. 109-114.

[12] Muto, S., Oshima, R., and Fujita, F., E., Acta Metall. 38 (1990) 685-694.

[13] Gunton, D. J., and Saunders, G. A., Sol. State Comm. 14 (1974) 865-868.

[14] Madhava, M. R., and Saunders, G. A., Phil. Mag. 36 (1977) 777-789.

[15] Zhou, X., MS Thesis, University of Maryland, 1991.

[16] Saxena, A., and Barsch, G.R., Physica D66 (1993) 195-204.

[17] Li, J., PhD. Thesis, University of Maryland, 1992. 